\title{
Application of Time-of-flight Near-infrared Spectroscopy to Detect Sugar and Acid Content in Satsuma Mandarin
}

\author{
Satoru Tsuchikawa, ${ }^{1}$ Emiko Sakai, and Kinuyo Inoue \\ Graduate School of Bioagricultural Sciences, Nagoya University, Nagoya 464-8601, Japan \\ Kumi Miyamoto \\ Wakayama Fruit Tree Experiment Station, Kibi, Wakayama 643-0022, Japan
}

AdDitional Index wORDs. time resolved profile, pulsed laser beam, soluble solid, free acid, refractive index, tissue

\begin{abstract}
Time-of-flight near infrared spectroscopy (TOF-NIRS) was applied to the detection of sugar and acid content in Satuma mandarin (Citrus unshiu Marc). The combined effects on the time resolved profiles of soluble solids, free acids, laser beam wavelength, and detection position of transmitted light were investigated in detail. Attenuance of peak maxima $\left(A_{t}\right)$ and time delay of peak maxima $(\Delta t)$ increased as sugar content increased. However, these optical parameters decreased with increases in acid content. In the case of a model sample of a polyurethane block immersed in specified solutions, $A_{t}$ and $\Delta t$ decreased by adding sucrose or citric acid. These results suggested that time resolved profile was governed not only by the concentration of soluble solid or free acid but also by other inherent factors of a fruit; for example, an optical characteristic of tissue or difference in refractive index between the tissue substance and the liquid. Results indicated the optimum optical parameters for detection of sugar and acid content were $A_{t}$ and $\Delta t$, respectively.
\end{abstract}

Satsuma mandarin (Citrus unshiu Marc) is the most popular horticultural crop in Japan with an annual production of $\approx 1.14$ million tons. However, the production itself is decreasing annually due to diversification of consumer-oriented food forcing the industry to focus on marketing the highest quality fruit. The selected approach has been to develop nondestructive technologies for quality determination and control. Nondestructive measurement systems that can accurately measure the internal quality simultaneously with minimal time and effort are highly desirable for marketing not only Satsuma mandarin, but also other agricultural commodities. Because the combination of soluble solids and free acids determines the taste of Satsuma mandarin, it is especially important to evaluate both.

In the past few decades, researchers have focused on the potential use of near infrared (NIR) spectroscopy, a practical spectroscopic procedure for the detection of organic compounds in organic matter (Burns and Ciurczak, 1992; Osborne, et al., 1993). The transmittance NIR method is more desirable for detecting internal information than the optical information from diffuse reflectance spectra which are confined to the subsurface layer of samples. We have already demonstrated the importance of NIR spectroscopy in the horticultural field and referred to several reports by other researchers (Tsuchikawa et al., 2002a). Also for Satsuma mandarin, several NIR techniques and devices using transmittance or reflectance have been proposed (Kawano et al., 1993; Miyamoto and Kitano, 1995; Miyamoto, 1998). Miyamoto and Kitano (1995) examined the applicability of NIR transmittance spectra as a nondestructive method for determining sugar and acid contents. They developed an on-line device, equipped with a halogen lamp and a linear-array silicon photodiode for grading fruit. With this device, sugar content could be evaluated with a standard error of prediction of $0.6 \%$, which fully satisfied both suppliers and consumers demands. However, acid content may be difficult to evaluate accurately because of its lower content of $\approx 1 \%$. Furthermore, behavior of transmitted light through an agricultural product is directly affected by physical and chemical

Received for publication 28 May 2002. Accepted for publication 4 Feb. 2003. ${ }^{1}$ Corresponding author; e-mail st3842@agr.nagoya-u.ac.jp. properties of the tissues, making it very important to examine in detail the optical characteristics of the tissue and the output origin of transmitted light.

Recently, we have developed a new optical system which precisely detected water core in apple (Tsuchikawa et al., 2002a). The system, made up of a parametric tunable laser and a NIR photoelectric multiplier, was based on the concept of time-offlight near infrared spectroscopy (TOF-NIRS), where a time resolved profile of transmitted output power was measured with nanosecond sensitivity (Tsuchikawa and Tsutsumi, 2002b). In the previous report (Tsuchikawa et al., 2002a) we investigated in detail the variation of time resolved profiles resulting from water-core tissue, laser beam wavelength and detection position of transmitted light. Light scattering state and substantial optical path length in apple were also estimated, which were key factors for the correct optical measurements. Results indicated that the optical parameter for detection of water core was the time delay of the peak maxima. Therefore, the intensity of output signal (e.g., absorbance) was not necessarily the best parameter for nondestructive determination.

Such a sophisticated optical technique should be available for other fruit. In this study, we applied TOF-NIRS to the detection of sugar and acid contents in Satsuma mandarin. The effect of these constituents on the transmitted output power was fully examined and used to estimate the optical parameter preferable for detecting internal quality. We also investigated the behavior of time resolved profile of a model sample (i.e., a polyurethane block immersed in several solutions) for simulation of the light scattering state in a fruit. These basic data can be helpful to realize an in-process measurement system.

\section{Material and Methods}

Measuring apparatus. The measuring system used was the same as that used in our previous report (Tsuchikawa et al., 2002b). A Q-switched Nd: YAG laser having an output energy of $60 \mathrm{~mJ} /$ pulse at $335 \mathrm{~nm}$, a pulse width of $5 \mathrm{~ns}$, a pulse repetition frequency of $10 \mathrm{~Hz}$, and a beam diameter of $6 \mathrm{~mm}$ was employed as the exciter laser. The wavelength $(\lambda)$ of the pulsed laser by 
optical parametric oscillation of a $\mathrm{BBO}\left(\beta-\mathrm{BaB}_{2} \mathrm{O}_{4}\right)$ crystal was tuned to $800,930,980$, and $1100 \mathrm{~nm}$, which corresponded to the vigorous light scattering region, the absorption band of sucrose, the absorption band of water, and the detectable upper limit of the output signal from an intact Satsuma mandarin, respectively. The monochromator was utilized to keep the spectral line width constant at $0.1 \mathrm{~nm}$. The transmitted output power from the sample was measured by a NIR photoelectric multiplier through a fiber optic cable having a diameter of $7 \mathrm{~mm}$. The fiber optic cable was directly in contact with the sample.

Time resolved profile refers to variation in intensity of the detected light beam with time. Combined effects on time resolved profiles of sugar content, acid content, laser beam wavelength $(\lambda)$, and detection position of transmitted light were investigated in detail. We focused on some typical parameters representing the variation in time resolved profiles. The normalized time resolved profiles of a Satsuma mandarin (68 $\mathrm{mm}$ in diameter) and its peel (thickness $=2 \mathrm{~mm}$ ) are illustrated in Fig. 1. The time resolved profile of the peel was employed as the reference of a zero value.

Variations in peak maxima and the time delay of peak maxima were examined under the experimental measuring conditions. The measure of attenuance $\left(A_{t}\right)$ was defined as follows:

$$
A_{t}=\operatorname{lng}\left(\frac{P w_{0}}{P w}\right)
$$

where $P w_{0}$ and $P w$ indicate the peak maxima of the reference and the object, respectively. Measured time delay of peak maxima $(\Delta t)$ was expressed as follows:

$$
\Delta t=t-t_{0}
$$

where $t_{0}$ and $t$ indicate the time at peak maxima of the reference and the object, respectively.

Fruit SAMPLES. The samples used were Satsuma mandarins harvested 5 Oct. to 20 Nov. 2001 from an orchard in Wakayama, Japan. The specific parameters tested in Satsuma mandarin are listed in Table 1. The concentration of soluble solids was measured with a digital refractometer(IPR 201,ATAGO Co., Tokyo, Japan), while the concentration of free acids was measured with a dual conductivity meter (WM-50EG, DKK-TOA Co., Tokyo, Japan). After the series of optical experiments, the pulp segments at an

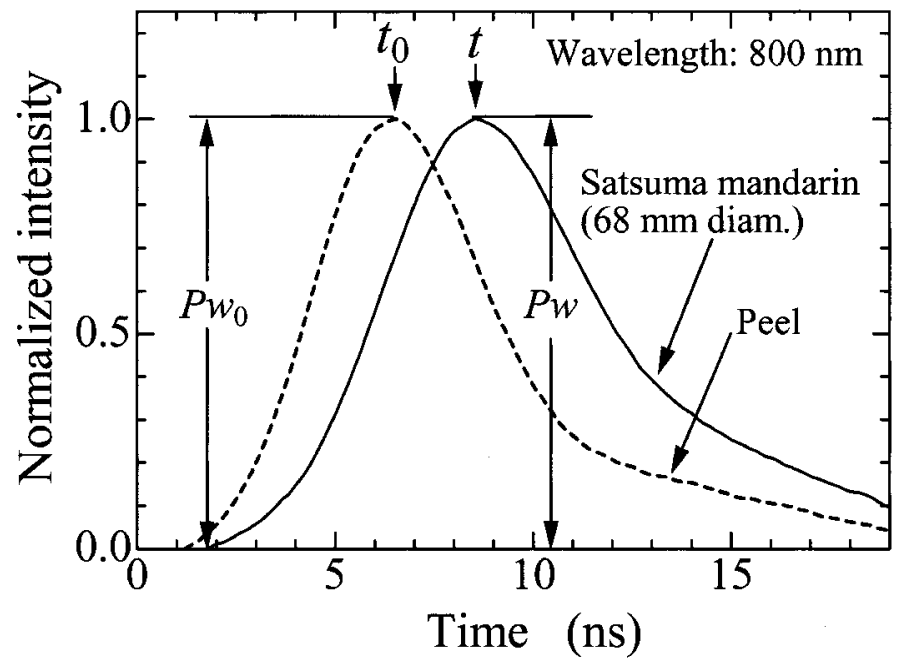

Fig. 1. Normalized time resolved profile of the transmitted output power. The equator of the Satsuma mandarin was irradiated vertically with a pulsed laser beam of $\lambda=800 \mathrm{~nm}$, and the transmitted output power was detected at side face. The solid and broken lines represent the profiles of the Satsuma mandarin and its peel (thickness $=2.0 \mathrm{~mm}$ ), respectively. irradiation and detection position were corrected for measurement of these constituents, the average of which was employed as the sugar and acid contents in a sample.

Model SAMPLES. We also measured the time resolved profile of a model sample for simulation of the light scattering state in a fruit. A polyurethane block $(50 \mathrm{~mm}$ wide, $50 \mathrm{~mm}$ high, and 20 $\mathrm{mm}$ in thick) was immersed in the specified liquid. Three solutions (water, $16.6 \%$ sucrose and $0.4 \%$ citric acid) were used. The model sample was inserted in the glass cell.

\section{Results and Discussion}

In this study, the time resolved profile was systematically measured. The equator of Satsuma mandarin was irradiated vertically with the pulsed laser, and the transmitted output power was detected at its opposite face and side face on the equator perpendicular to irradiation direction as illustrated in Fig. 2. The sampling time for one pulsed laser was $100 \mathrm{~ns}$, and 300 scans were co-added in order to improve the signal-to-noise ratio. Each sample was measured five times within a given detection position and wavelength of the laser beam. During the measurement periods, no significant changes in time resolved profile were observed. The following results are therefore presented as the average signal of five measurements.

VARIATIONS OF OPTICAL PARAMETERS WITH SUGAR AND ACID CONTENTS In Satsuma Mandarin. Since the time resolved profile was strongly affected by the sample size (Tsuchikawa, et al., 2002a), $A_{t}$ and $\Delta t$ were standardized by dividing the distance between irradiation position and detection position $l$ (Fig. 2). Fig. 3 shows the variation of $A_{t}$ or $\Delta t$ standardized by dividing $l$ with the sugar or acid content in Satsuma mandarin. A pulsed laser beam of $\lambda=980 \mathrm{~nm}$ irradiated the sample, and the transmitted output power was detected at its side face. Both $A_{t} / l$ and $\Delta t / l$ increased as sugar content increased, which could be expressed under the level of significance at $2 \%$. These results mean that the intensity of transmitted light decreased and the optical path length increased incrementally with sugar content. On the contrary, $\Delta t / l$ decreased as acid content increased under the same level of significance (optical path length decreased inversely with acid content). We could not find a significant correlation between $A_{t} / l$ and acid content. Therefore, time resolved profile of Satsuma mandarin varied characteristically with soluble solids and free acids.

These results were observed independently from the pulsed laser wavelength; however, the correlation coefficient between sugar or acid content and optical parameters varied with the wavelength. We could not find a significant relationship between the optical parameters detected at an opposite face and the constituents in Satsuma mandarin. This may result from the detection of straight or near-straight transmitted beams, which do not include the useful information of an optically thin sample (Leonardi and Burns, 1999a, 1999b). On the other hand, the time resolved profile at the side face of fruit reflects the characteristics of scattered light that might include the chemical information.

Relationships between optical Parameters and Sugar CONTENT UNDER LIMITED ACID CONTENT DEVIATION. Although the time resolved profile varied with sugar and acid contents, their characteristics were entirely different from each other. It was therefore necessary to examine the relationship between optical parameters and these constituents under the known conditions. As a first step, we investigated the relationship between time resolved profile and sugar content under limited acid content deviation. Figure 4 shows the variations of $A_{t} / l$ and $\Delta t / l$ with sugar content 


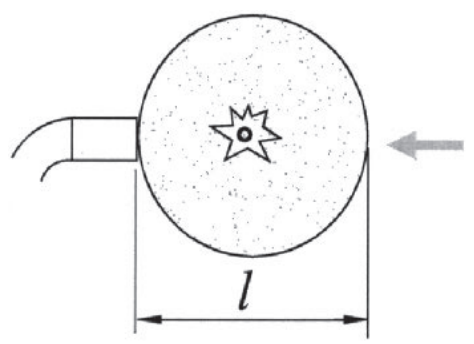

Pattern A

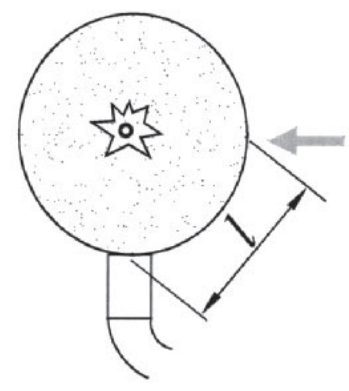

Pattern B
Fig. 2. Illustration of the measurement arrangement.

in Satsuma mandarin under limited acid content deviation in 19 selected samples. A pulsed laser beam of $\lambda=980 \mathrm{~nm}$ irradiated the sample, and the transmitted output power was detected at its side face. As expected, this limitation of free acid deviation resulted in a high correlation between optical parameters and sugar content compared to Fig. 3. The correlation coefficients between sugar content and $A_{t} / l$ or $\Delta t / l$ are summarized in Fig. 5. For each sample, there were no data at $\lambda=980 \mathrm{~nm}$ for the opposite face because of instability of the output signal. $A_{t} / l$ showed relatively high correlation with sugar content independent of the wavelength or the detection position. On the other hand, the relationship between sugar content and $\Delta t / l$ was statistically significant only at restricted wavelengths. Furthermore, we could not find a significant correlation coefficient in the case of detection at the opposite face. These results suggest that $A_{t}$ or the intensity of transmitted light is a suitable optical parameter for evaluation of sugar content.

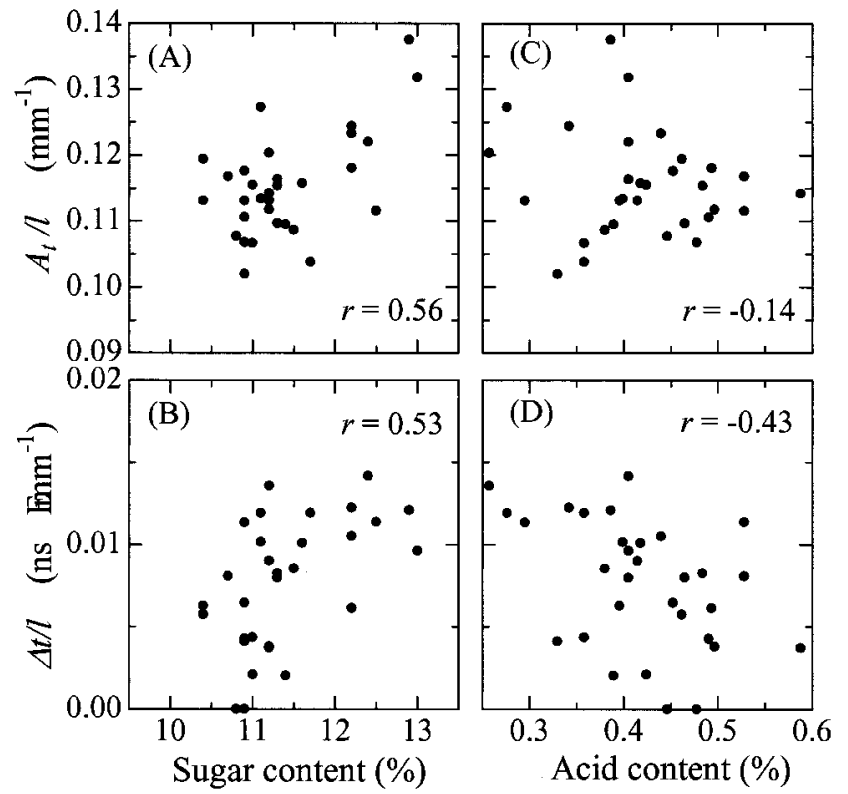

Fig. 3. Relationships between sugar or acid content and optical parameters. (A) Sugar content $(\%)$ vs. attenuance $\left(A_{t}\right)$ standardized by dividing $l,(\mathbf{B})$ sugar content vs. time delay of peak maxima $(\Delta t)$ standardized by dividing $l,(\mathbf{C})$ acid content $(\%)$ vs. $A_{\ell} /$, and (D) acid content vs. $\Delta t / l$. The equator of the Satsuma mandarin was irradiated vertically with a pulsed laser beam of $\lambda=980 \mathrm{~nm}$, and the transmitted output power was detected at its side face on the equator perpendicular to irradiation direction. The distance between the irradiation position and the detection position $(l)$ was varied $39.6 \mathrm{~mm}$ to $53.0 \mathrm{~mm}$ (see Table 1 and Fig. 2). Sugar content varied from $10.4 \%$ to $13.0 \%$, and acid content also varied from $0.26 \%$ to $0.59 \%$, respectively (also see Table 1 ). Each symbol respects the mean of five observations. $r$ indicates the correlation coefficient.
This also supports the usefulness of a NIR technique for quality detection as reported by several researchers (Kawano et al., 1993; Miyamoto and Kitano, 1995; Miyamoto, 1998).

RELATIONSHIPS BETWEEN OPTICAL PARAMETERS AND ACID CONTENT UNDER LIMITED SUGAR CONTENT DEVIATION. Next, we examined the relationship between time resolved profile and acid content under limited sugar content. Figure 6 shows the variations in $A_{t} / l$ and $\Delta t / l$ with acid content in Satsuma mandarin under limited sugar content among 15 selected samples. A pulsed laser beam of $\lambda=980 \mathrm{~nm}$ irradiated the sample, and the transmitted output power was detected at its side face. Also in this case, the limitation of soluble solids contributed to the improvement of the correlation between acid content and the optical parameter for time resolved profile. The correlation coefficients between acid content and $A_{t} / l$ or $\Delta t / l$ are summarized in Fig. 7 . We could not find a significant relationship between acid content and either of the optical parameters except the time resolved profile at $980 \mathrm{~nm}$ on the side face, whereas sugar content deviation was considerably limited by selecting the sample.

Acid content in Satsuma mandarin usually ranges from $0.5 \%$ to $1.5 \%$ (Ting, 1969), thus validation using a commercial spectrophotometer based on normal NIR methods should not be expected to have high accuracy. Although detection of acid content by TOF-NIRS should be limited, our results suggested that time resolved profile at $980 \mathrm{~nm}$ detected on the side face provided useful information. The measure of time delay of peak maxima $\Delta t$ may be an optimum optical parameter, which was also a suitable parameter for detecting water core in apples (Tsuchikawa et al., 2002b).

SimUlation OF LIGHT SCATTERING STATE IN A FRUIT BY MODEL SAMPLE. The reason the time resolved profile functioned inversely for the variation in sugar and acid contents in Satsuma mandarin is not explainable from our experimental results. Akey to consider is the effect of cellular structure in a fruit on the time resolved profile based on the difference in the refractive index between tissue and soluble solids or free acids. We systematically measured the time resolved profile of a model sample which could represent the relatively isotropic light scattering state in a fruit.

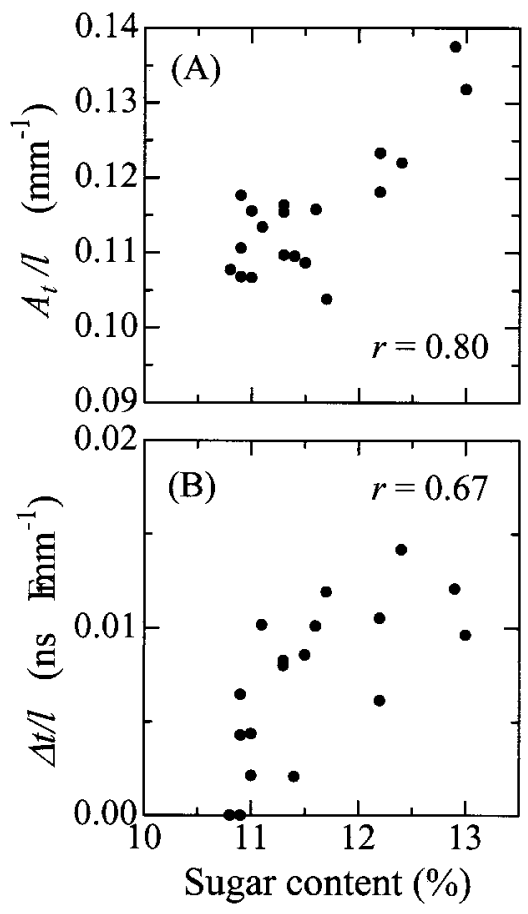

Figure 8 shows the time resolved profiles of transmitted output power

Fig. 4. Relationships between optical parameters standardized by dividing $l$ and sugar content.(A) Sugar content (\%) vs. attenuance $\left(A_{t} l\right)$, and (B) sugar content vs. time delay of peak maxima $(\Delta t / l)$. Nineteen samples were selected where an acid content was limited in the range of $0.45 \%$ to $0.55 \%$, so that sugar content varied from $10.8 \%$ to $13.0 \%$. The equator of the Satsuma mandarin was irradiated vertically with a pulsed laser beam of $\lambda=980 \mathrm{~nm}$, and the transmitted output power was detected at its side face on the equator perpendicular to irradiation direction. The distance between the irradiation position and the detection position $(l)$ was varied $40 \mathrm{~mm}$ to $49 \mathrm{~mm}$. Each symbol respects the mean of five observations. $r$ indicates the correlation coefficient. 


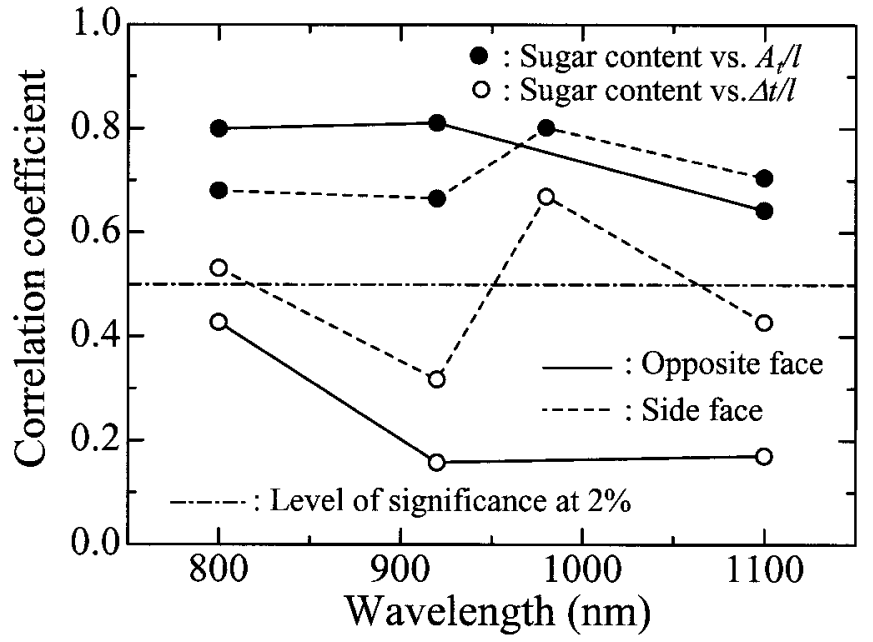

Fig. 5. Correlation coefficients between sugar content under the limited acid content deviation and attenuance $\left(A_{t} / l\right)$ or time delay of peak maxima $(\Delta t / l)$. Nineteen samples were selected where an acid content was limited in the range of $0.45 \%$ to $0.55 \%$, so that sugar content varied from $10.8 \%$ to $13.0 \%$. The equator of the Satsuma mandarin was irradiated vertically. The solid and open circles indicate the correlation coefficients for sugar content vs. $A / l$ and sugar contents vs. $\Delta t / l$, respectively. The solid and broken lines express the results at the detection position at opposite face and side face to irradiation position, respectively. The dash-dotted line indicates the level of significance at $2 \%$. The distance between the irradiation position and the detection position $(l)$ was varied 40 to $49 \mathrm{~mm}$. Each symbol respects the mean of five observations.

of the model sample (the polyurethane block immersed in three liquids). The output signal increased and its peak maxima was increased by adding either sucrose or citric acid. That is, the polyurethane block immersed in the solution of known concentration would transmit much more energy under the condition of less light scattering. This may result from the difference in refractive index $(n)$ between the polyurethane block and the liquid. The $n$ of the polyurethane block should be over 1.5. On the other hand, $n$ of water, solution of citric acid, and solution of sucrose were, $1.333,1.339$, and 1.366, respectively. Light scattering in the model
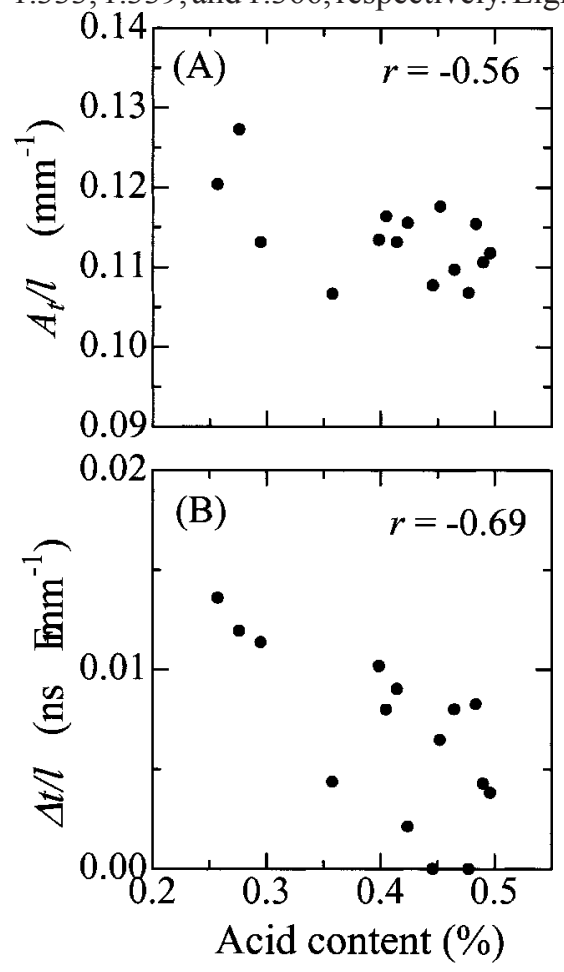

sample should decrease as the difference in $n$ between the polyurethane block and the liquid de-

Fig. 6. Relationships between optical param-eters standardized bydividing $l$ andacidcontent.(A) Acidcontentvs. attenuance $(A / l)$, and(B)acidcontentvs.timedelay of peak maxima $(\Delta t / l)$. Fifteen samples were selected where the sugar content was limited in the range of $10.8 \%$ to $11.3 \%$, so that acid content varied from $0.26 \%$ to $0.50 \%$. The equator of the Satsuma mandarin was irradiatedverticallywithapulsed laser beam of $\lambda=980 \mathrm{~nm}$, and the transmitted output power was detec-ted at its side face on the equator perpendicular to irradiationdirection. Thedistance between the irradiation position and the detection position $(l)$ was varied 42 to $53 \mathrm{~mm}$. Each symbol respects the mean of five observations. $r$ indicates the correlation coefficient. creases, so that these results are reasonable from the viewpoint of geometric optics (Kortüm, 1969).

Figure 9 shows the variation of $A_{t}$ and $\Delta t$ per unit concentration of sucrose or citric acid. As determined from this figure, variations in $A_{t}$ and $\Delta t$ for citric acid were larger than those for sucrose, independent of the wavelength. The variation in $\Delta t$ at $\lambda=$ $930 \mathrm{~nm}$ and $980 \mathrm{~nm}$ for citric acid was extremely large; however, this result could not be simply expressed from the difference in refractive indices. Variation in refractive index for sucrose per unit concentration is much larger than that for citric acid. Therefore, other factors [i.e., variation in thermal motion of water molecule with the dissolved substance (Uedaira, 1998)] will relate strongly to these phenomena. In any case, the optical unity between the solid (i.e., the fruit tissue or the polyurethane block) and the solution would contribute less light scattering.

Finally, we will summarize the experimental results to explain consistently the light absorption-scattering state in a fruit. Output signal from the model sample increased (i.e., $A_{t}$ decreased) as sugar content increased, while time delay of peak maxima, $\Delta t$, decreased with similar sugar increments. However, time resolved profile for Satsuma mandarin showed an inverse phenomenon (i.e., $A t$ and $\Delta t$ increased as sugar content increased). As an example, the time resolved profiles with different sugar contents are depicted in Fig. 10A, where citric acid and sample size are of the same value. We explain such paradoxical phenomena by introducing the following hypothesis: the optical characteristic of the tissue changed with increasing sugar content so that the optical path length increased (i.e., increased viscosity of fruit juice by decomposition of cellulose). In this case, the effect of refractive index on time resolved profile as shown in Figs. 8 and 9 may be canceled.

On the contrary, an increase in output signal and a decline in $\Delta t$ were also observed for an increase in citric acid in the model sample. The time resolved profile for Satsuma mandarin showed the same phenomena only at the restricted wavelength. As an example, the time resolved profiles with different acid contents are

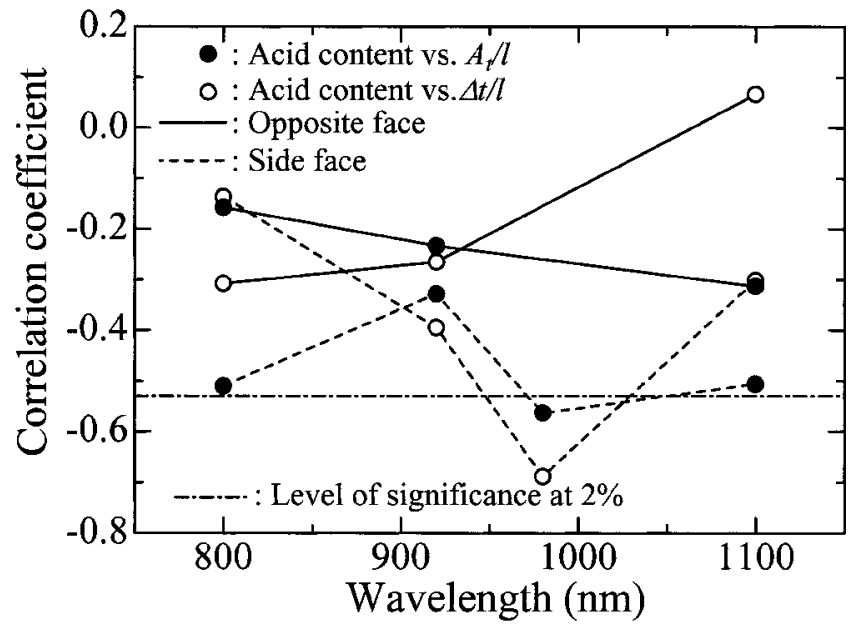

Fig. 7. Correlation coefficients between acid content under the limited sugar content deviation and attenuance $\left(A_{t} / l\right)$ or time delay of peak maxima $(\Delta t / l)$. Fifteen samples were selected where the sugar content was limited in the range of $10.8 \%$ to $11.3 \%$, so that acid content varied from $0.26 \%$ to $0.50 \%$. The equatorof the Satsumamandarin was irradiated vertically. The solid and open circles indicate the correlation coefficients for acid content vs. $A_{\ell} /$ and acid content vs. $\Delta t / l$, respectively. The solid and broken lines express the results at the detection position at opposite face and side face to irradiation position, respectively. The dash-dotted line indicates the level of significance at $2 \%$. The distance between the irradiation position and the detection position $(l)$ was varied 42 to $53 \mathrm{~mm}$. Each symbol respects the mean of five observations. 


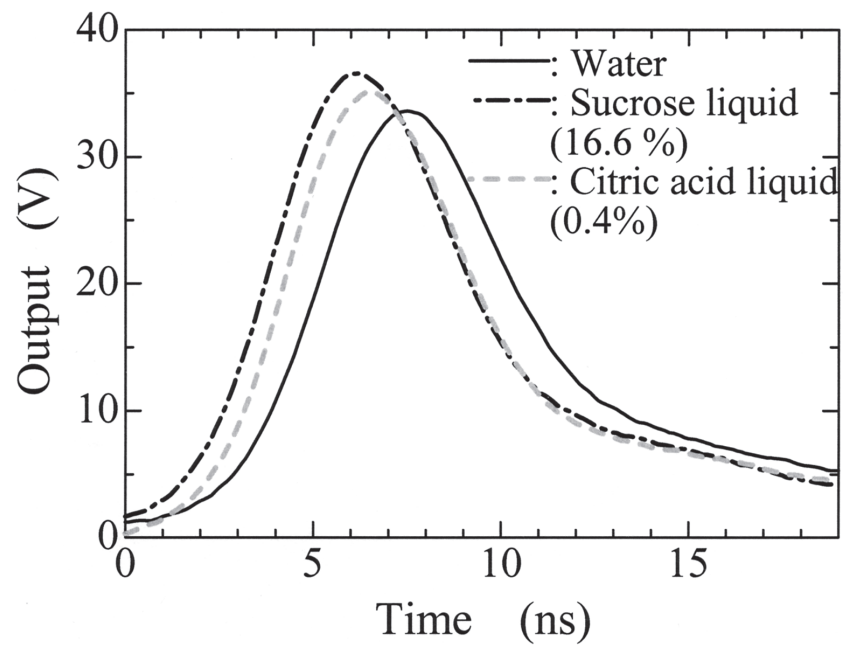

Fig. 8. Time resolved profile of the transmitted output power for the model sample (the polyurethane block immersed in several liquids). The center of the sample was irradiated vertically with a pulsed laser beam of $\lambda=980 \mathrm{~nm}$, and the transmitted output power was detected at its opposite face. The solid, broken, and dash-dotted lines represent the profiles of the model sample immersed in water, solution of sucrose in concentration of $16.6 \%$, and solution of citric acid in concentration of $0.4 \%$, respectively. The output was represented as an original signal of voltage.

depicted in Fig. 9B, where sugar content and sample size are of constant value. In this case, the effect of optical unity between the solid and the liquid on the time resolved profile would be dominant over that resulting from the variation in tissue, which functions inversely in the behavior of $A_{t}$ and $\Delta t$. This could be estimated from Fig. 9, where variation of $A_{t}$ and $\Delta t$ per unit concentration of citric acid was extremely large. Since Satsuma mandarin contains small amounts of citric acid, the relationship between the tissues and the concentration of solution should very sensitive, so that the trend of $A_{t}$ and $\Delta t$ will not be necessarily determined with statistical significance at every wavelength.

Figure 11 outlines of these new concepts. An increase in soluble solids or free acids causes an increase in $A_{t}$ and $\Delta t$ from the standpoint of variation of tissue, while it leads to a decrease in optical parameters from the standpoint of variation in refractive index. Therefore, this figure explains visually how output patterns between the constituents of a fruit and the optical parameters were determined by the superior (or inferior) effect of tissue (or refractive index) on the time resolved profile. In this way, time resolved profile was governed not only by the concentration of soluble solids or free acids but also other inherent factors of a fruit (e.g., optical characteristics of a tissue or differences in the refractive index between the tissue and liquid). A series of basic data and new concepts indicated the applicability of TOF-NIRS to an in-process measurement system for practical use by the Satsuma mandarin industry.

\section{Literature Cited}

Burns, D.A. andE.W. Ciurczak. 1992. Handbook of near-infrared analysis. Marcel Dekker, New York.

Kawano, S., T. Fujiwara, and M. Iwamot. 1993. Nondestructive determination of sugar contents in Satsuma mandarin using near infrared transmittance. J. Jpn. Soc. Hort. Sci. 62:465-470.

Kortüm, G. 1969. Reflectance spectroscopy. Springer-Verlag, New York.

Leonardi, L. and D.H. Burns. 1999a. Quantitative measurements in scattering media: Photon time-of-flight analysis with analytical descriptions. Appl. Spectroscopy 53:628-636.

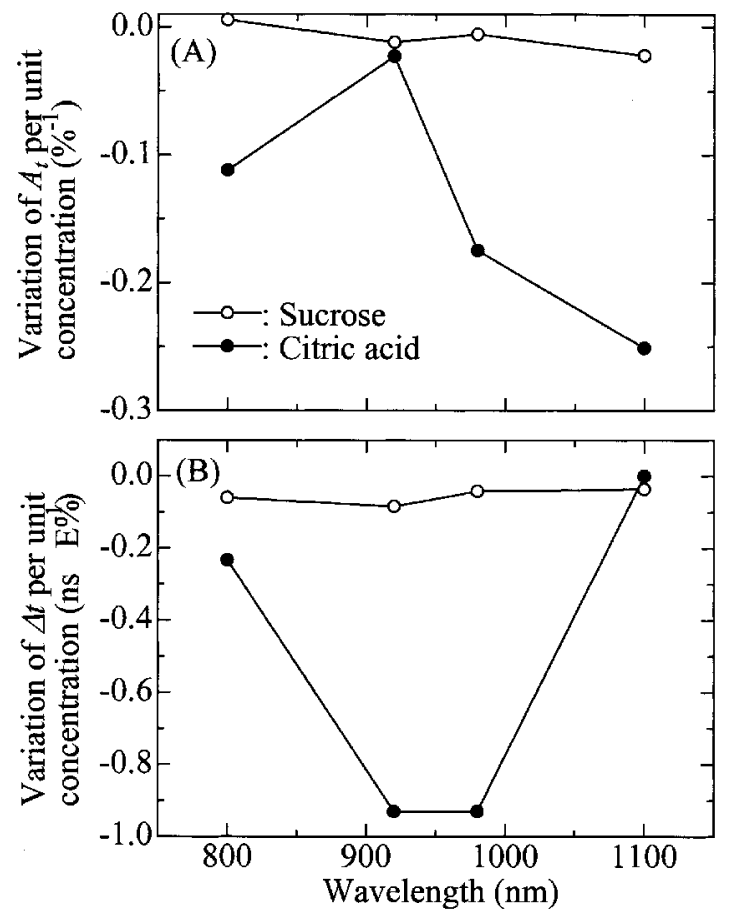

Fig. 9. Variation of optical parameters with solution of sucrose or citric acid in the model sample at each wavelength. (A) Attenuance $\left(A_{t}\right)$ and $(\mathbf{B})$ time delay of peak maxima $(\Delta t)$. The center of the samplewas irradiated vertically, and the transmitted outputpower was detected at its opposite face. The time resolved profile of water was employed as the reference of a zero value (see Fig. 8). The open and solid circles indicate the measured values per unit concentration of sucrose and citric acid, respectively. Each symbol respects the mean of five observations. Legend in A applies to B.
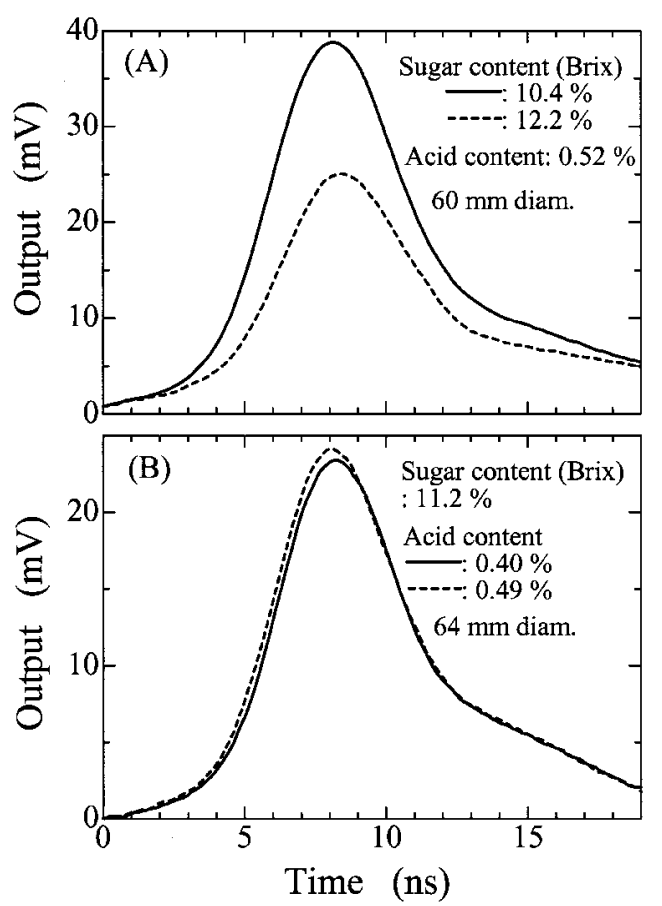

Fig. 10. Time resolved profiles of transmitted output power for Satsuma mandarin. (A) Sugar contents in samples (\%) indicated by the solid and broken lines are $10.4 \%$ and $12.2 \%$, respectively. Citric acid $(0.52 \%)$ and sample size (diam-eter: $60 \mathrm{~mm}$ ) are same value. (B) Acid contents in samples indicated by the solid and broken line are $0.40 \%$ and $0.49 \%$, respectively. Sugar content $(11.2 \%)$ and sample size (diam-eter: $64 \mathrm{~mm}$ ) are same value. The equator of the Satsuma mandarin was irradiated verti-cally with a pulsed laser beam of $\lambda=980 \mathrm{~nm}$, and the transmitted output power was detected at its side face perpendicular to irradiation direction. 


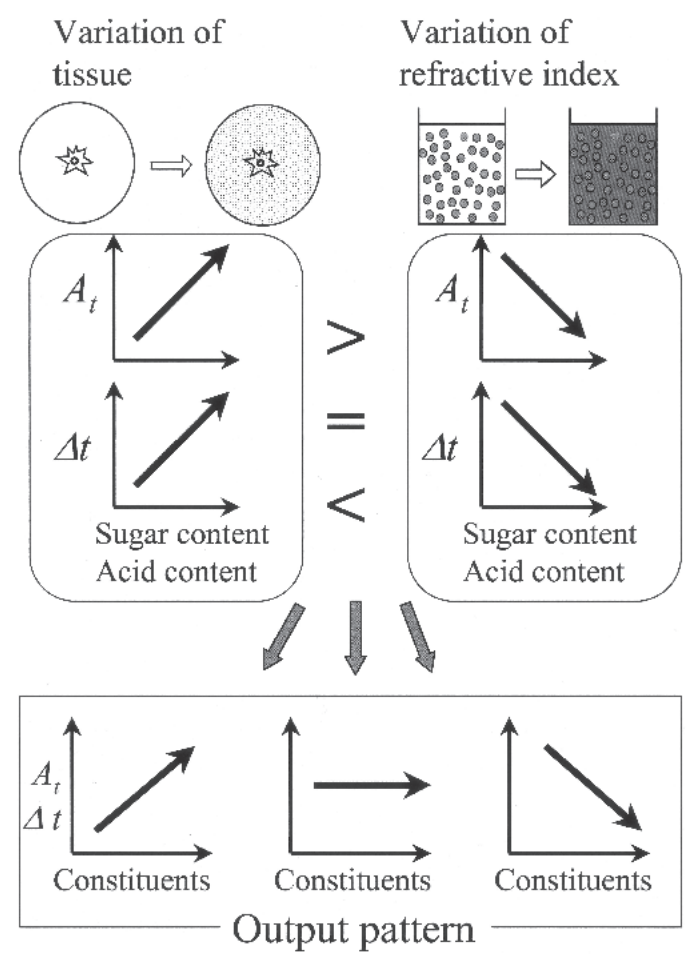

Leonardi, L. and D.H. Burns. 1999b. Quantitative mutiwavelength constituent measurements using single-wavelength photon time-of-flight correction. Appl. Spectroscopy 53:637-646.

Miyamoto, K. and Y. Kitano. 1995. Non-destructive determination of sugar content in Satsuma mandarin fruit by near infrared transmittance spectroscopy. J. Near Infrared Spectroscopy 3:227-237.

Miyamoto, K. 1998. Internal quality control of citrus frit in a packing house using near infrared spectroscopy. J. Near Infrared Spectroscopy 5:127-137.

Osborne, B.G., T. Fearn, and P.H. Hindle. 1993. Practical NIR spectroscopy with applications in food and beverage analysis. Longman Sci. and Technol., Harlow, U.K.

Ting, S.V. 1969. Distribution of soluble components and quality factors in the edible portion of citrus fruits. J. Amer. Soc. Hort. Sci. 94: $515-519$.

Tsuchikawa, S., S. Kumada, K. Inoue, and R. Cho. 2002a. Application of time-of-flight near-infrared spectroscopy for detecting water core in apples. J. Amer. Soc. Hort. Sci. 127:303-308.

Tsuchikawa, S. and S. Tsutsumi. 2002b. Application of time-of-flight near infrared spectroscopy to wood with anisotropic cellular structure. Appl. Spectroscopy 56:1117-1124.

Uedaira, H. 1998. What is water? (in Japanese) Kodansha, Tokyo.

Fig. 11. Outline of new concepts for consistent explanation of the light absorptionscattering state in a fruit. 